\title{
Low-Cost and Highly Sensitive Liquid Refractive Index Sensor Based on Polymer Horizontal Slot Waveguide
}

\author{
Xiaoxia MA*, Kaixin CHEN, Jieyun WU, and Lingfang WANG \\ School of Optoelectronic Science and Engineering, University of Electronic Science and Technology of China, Chengdu \\ 611731, China \\ ${ }^{*}$ Corresponding author: Xiaoxia MA E-mail: maxiaoxia_cd@163.com
}

\begin{abstract}
We analyze and explore the potential of using a polymer horizontal slot waveguide as light-analyte interactive region to implement a low-cost and highly sensitive liquid refractive index sensor. Numerical analysis shows that the optimized polymer horizontal slot waveguide is able to realize high waveguide sensitivity. With the optimized horizontal slot waveguide, polymer liquid refractive index sensors based on Mach-Zehnder interferometer (MZI) and microring resonator (MRR) are then investigated numerically, and the results show that the MZI-based sensor can achieve high sensitivity of $17024 \mathrm{~nm} /$ RIU and low limit of detection (LOD) of $1.76 \times 10^{-6}$ RIU while the MRR-based sensor can achieve the sensitivity of $177 \mathrm{~nm} / \mathrm{RIU}$ and the LOD of $1.69 \times 10^{-4}$ RIU with a very small footprint. Compared with the sensors employing conventional silicon or silicon nitride vertical slot waveguide, the sensors employing polymer horizontal slot waveguide exhibit comparable performances but simpler and lower fabrication costs.
\end{abstract}

Keywords: Integrated optics devices; polymer waveguides; sensors; waveguides

Citation: Xiaoxia MA, Kaixin CHEN, Jieyun WU, and Lingfang WANG, "Low-Cost and Highly Sensitive Liquid Refractive Index Sensor Based on Polymer Horizontal Slot Waveguide," Photonic Sensors, 2020, 10(1): 7-15.

\section{Introduction}

Recently, a novel structure called slot waveguide has attracted great interest due to its remarkable characteristics to guide and confine light in a nanometric low index material region. A slot waveguide consists of two strip waveguide cores formed with high index material separated by a subwavelength slot region of low index material. Its operating principle is based on the discontinuity of electric field at the high-index-contrast interface. When the dimension of the slot is smaller than the decay length of the field from the interface into the low-index region, the electric field remains high within the slot, as a result, the optical intensity in the slot is much higher than that in the high-index cores $[1,2]$. Thus, compared with the conventional waveguide, using the slot waveguide as sensing region can achieve stronger light-analyte interaction, which means a significant enhancement in sensing sensitivity. In view of all the advantages above, the slot waveguide is very attractive for sensing applications [3].

Up to now, various slot waveguide sensors have been demonstrated. Typically, a slot-waveguide microring resonator (MRR) fabricated on a $\mathrm{Si}_{3} \mathrm{~N}_{4}-\mathrm{SiO}_{2}$ platform working as a biochemical sensor was firstly demonstrated experimentally, and the sensitivity of $212 \mathrm{~nm} /$ refractive index units (RIU) was observed [4]. A slot-waveguide ring resonator

Received: 7 January 2019 / Revised: 21 April 2019

(C) The Author(s) 2019. This article is published with open access at Springerlink.com

DOI: $10.1007 / \mathrm{s} 13320-019-0560-y$

Article type: Regular 
on silicon on insulator (SOI) was also explored for label-free sensing of proteins [5]. Subsequently, a Mach-Zehnder interferometer (MZI) biosensor based on $\mathrm{Si}_{3} \mathrm{~N}_{4}$ slot waveguide was also demonstrated, and the sensitivity of $1864 \pi$ /RIU was obtained with $7 \mathrm{~mm}$ long slot waveguide sensing arm [6]. While a refractive index sensor based on polymer slot waveguide Young interferometer was developed in [7], a refractive index difference of $6.4 \times 10^{-6}$ RIU was experimentally observed. In addition, Bragg gratings [8] and directional coupler [9] employing slot waveguide were also proposed for sensing applications.

There are two types slot waveguides based on the orientation of slot, namely vertical and horizontal slot waveguides. For the vertical slot waveguides, the narrow slot produced by carefully etching usually requires expensive electron beam lithography and inductively coupled plasma etching equipment [10]. Moreover, etching such a narrow vertical slot usually results in a slant sidewall with large roughness, and hence increases the scattering loss [11] and limits the detection sensitivity [12]. Fortunately, these problems can be avoided by using horizontal slot waveguides [13, 14]. The horizontal slot waveguides can be fabricated layer by layer and therefore, the slot height can be controlled precisely and smooth interfaces can be realized easily, resulting in low scattering loss in interfaces [15]. Furthermore, the horizontally penetrated slot provides a better fluidic channel and thus better infiltration compared with the vertical slot [16]. In view of the advantages above, optical sensing employing the horizontal slot waveguide as light-analyte interactive region has been explored in [13-16].

For these reported sensors based on the horizontal slot waveguides, all of them are explored with high refractive index materials such as silicon nitride [14] or SOI [13, 15, 16] by using the costly method of chemical vapor deposition. However, in the sensor applications, reducing the cost of the devices is desired so as to provide a potential use of disposable sensors [17]. Polymer has been proved to be a promising material for fabricating optical waveguide sensors due to its attractive properties, including low optical loss, transparency in a wide wavelength range, low fabrication costs, compatibility with various substrates, and easy integration with other devices $[17,18]$. In addition, from a fabrication point of view, polymer is a very promising material platform for the implementation of a horizontal slot waveguide sensor because multilayer structures can be easily realized by the spin-coating technique with polymer materials. Optical sensing based on the conventional polymer optical waveguide [17-19] and polymer vertical slot waveguide $[7,20]$ has been explored so far. However, to the best of our knowledge, there is no previous report on the exploration of optical sensing based on polymer horizontal slot waveguides.

In this paper, the potential for using a polymer horizontal slot waveguide as light-analyte interactive region to implement a low-cost and highly sensitive liquid refractive index sensor is explored. Numerical analysis reveals that with the optimized geometrical parameters, the designed polymer horizontal slot waveguide can achieve high waveguide sensitivity. Our designed MZI- and MRR-based liquid refractive index sensors with the optimized polymer horizontal slot waveguide exhibit comparable performances by virtue of their sensitivity of $17024 \mathrm{~nm} / \mathrm{RIU}$ and $177 \mathrm{~nm} / \mathrm{RIU}$ and the LOD of $1.76 \times 10^{-6}$ RIU and $1.69 \times 10^{-4}$ RIU, respectively, but simpler and lower fabrication costs in comparison with the sensors employing conventional silicon or silicon nitride vertical slot waveguide.

\section{Polymer horizontal slot waveguide}

\subsection{Schematic of the polymer horizontal slot waveguide}

The three-dimensional (3-D) view of the proposed polymer horizontal slot waveguide is shown schematically in Fig. 1(a). In sensing region, 
it consists of two cores formed with polymer and separated in the vertical direction by a horizontal thin slot having a much lower refractive index compared with the cores, while in other regions it consists of only one core. The upper core is supported by its two ends and lateral beams, as shown in Fig. 1(a). With such a waveguide configuration, there is a discontinuity of the electric field along the $y$ direction on the top and bottom interfaces of the slot, which results in the transvers magnetic (TM) mode that has much higher amplitude in the slot than that in the cores. Thus, the light can be enhanced and confined in the slot region for the TM mode, and only the fundamental TM mode is involved in the following design and analysis work.

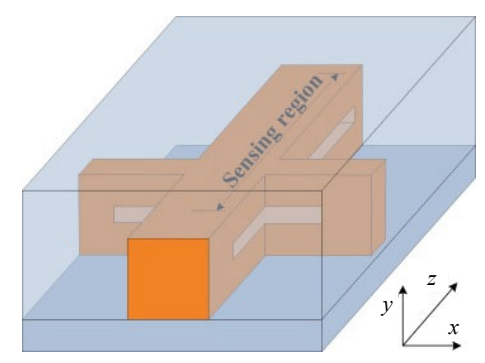

(a)

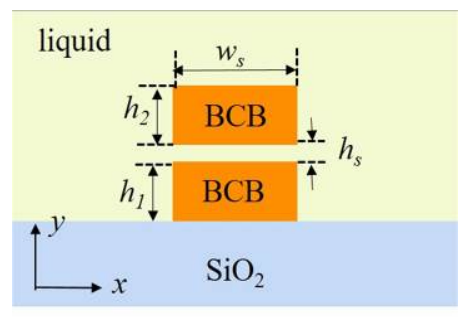

(b)

Fig. 1. 3-D view (a) and cross-sectional view (b) of the proposed polymer horizontal slot waveguide.

The cross-sectional view of the slot waveguide together with the materials used in this work is shown schematically in Fig. 1(b). The core is the polymer benzocyclobutene (BCB), the substrate is silica $\left(\mathrm{SiO}_{2}\right)$, the cladding is liquid analyte to be tested, and the slot is assumed to be completely filled with the liquid analyte. The refractive indices of $\mathrm{BCB}$ and $\mathrm{SiO}_{2}$ are 1.5370 and 1.4444 for the TM polarization, measured with a commercial prism coupler at $1538 \mathrm{~nm}$, and both have negligible material dispersion in the $\mathrm{C}$ band. The slot waveguide has the identical width of $w_{s}$ but different heights of the bottom and top waveguides of $h_{1}$ and $h_{2}$, respectively. The height of the horizontal slot is $h_{s}$.

\subsection{Waveguide investigation}

The waveguide sensitivity $S_{w}$ represents the overlap of the mode field of the waveguide with the liquid analyte and is measured as a change in the effective refractive index $n_{\text {eff }}$ of the waveguide mode upon the change in the refractive index $n$ of the liquid analyte:

$$
S_{w}=\partial n_{\text {eff }} / \partial n
$$

where $S_{w}$ is an extremely important parameter of the waveguide in sensing applications, which is vital to achieve a maximum sensitivity $S$ of the device.

To increase the value of $S_{w}$, we need to optimize the geometrical parameters of the slot waveguide to achieve a maximum effective index variation $\Delta n_{\text {eff }}$ with $n$ in the range from 1.3180 to 1.3204 , which corresponds to the refractive index of deionized water and $2 \%(\mathrm{~mol} / \mathrm{L})$ glucose liquid at the operating wavelength of $1538 \mathrm{~nm}$ [21, 22]. Firstly, for this purpose, we investigate the change of $\Delta n_{\text {eff }}$ with the liquid refractive index change $\Delta n$ for different slot waveguide height $h\left(h=h_{1}+h_{2}+h_{s}\right)$ with a commercial mode solver (COMSOL) at an ambient temperature of $22{ }^{\circ} \mathrm{C}$ by assuming the height difference $\Delta h=h_{2}-h_{1}=200 \mathrm{~nm}, h_{s}=140 \mathrm{~nm}$, and $w_{s}=2.0 \mu \mathrm{m}$. Note that the heights of the upper and the lower waveguide cores are set to be different considering that the refractive index of the $\mathrm{SiO}_{2}$ substrate is larger than that of the liquid cladding. The calculated results are shown in Fig. 2(a), which clearly indicates that $\Delta n_{\text {eff }}$ has a maximum value for the same $\Delta n$ at $h=1.7 \mu \mathrm{m}$, corresponding to $h_{1}+h_{2}=$ $1.56 \mu \mathrm{m}$. Thus, the total height of the two cores is set to be $h_{1}+h_{2}=1.56 \mu \mathrm{m}$ in the following design work. Secondly, to find an optimum value of $h_{s}$, we calculate the variation of $\Delta n_{\text {eff }}$ with $\Delta n$ for the $h_{s}$ 
changing from $140 \mathrm{~nm}$ to $290 \mathrm{~nm}$ with an increment of $30 \mathrm{~nm}$ while keeping other parameters unchanged. The results are shown in Fig. 2(b), which indicates that, for a certain change of $n, \Delta n_{\text {eff }}$ increases firstly and then decreases with an increase in $h_{s}$ and

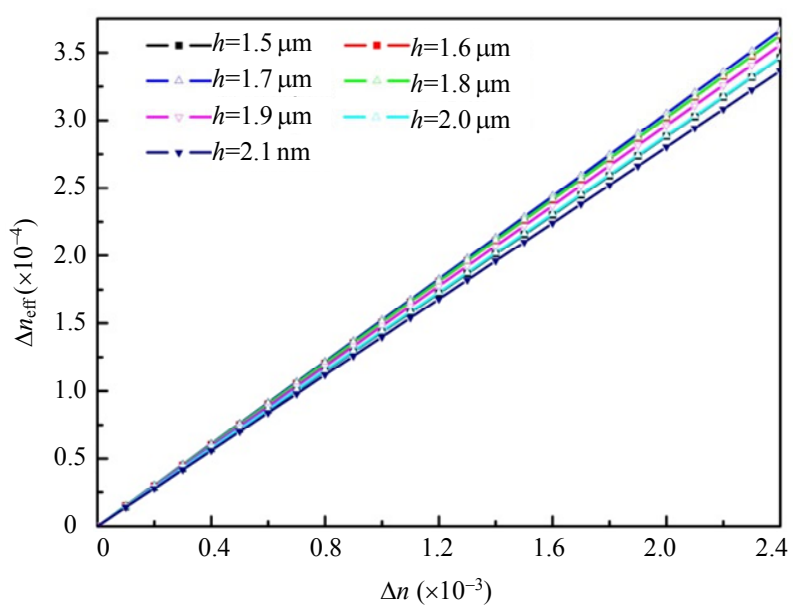

(a)

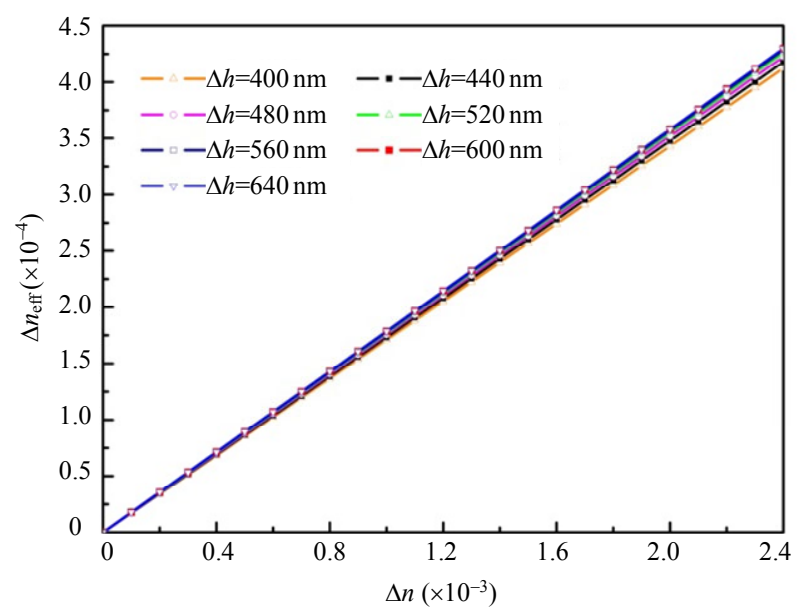

(c) achieves a maximum value when $h_{s}=200 \mathrm{~nm}$. This is because much more of the mode field, at a smaller $h_{s}$, is leaked into the substrate, while at a larger $h_{s}$, it is confined in the waveguide core compared with a modest $h_{s}$.

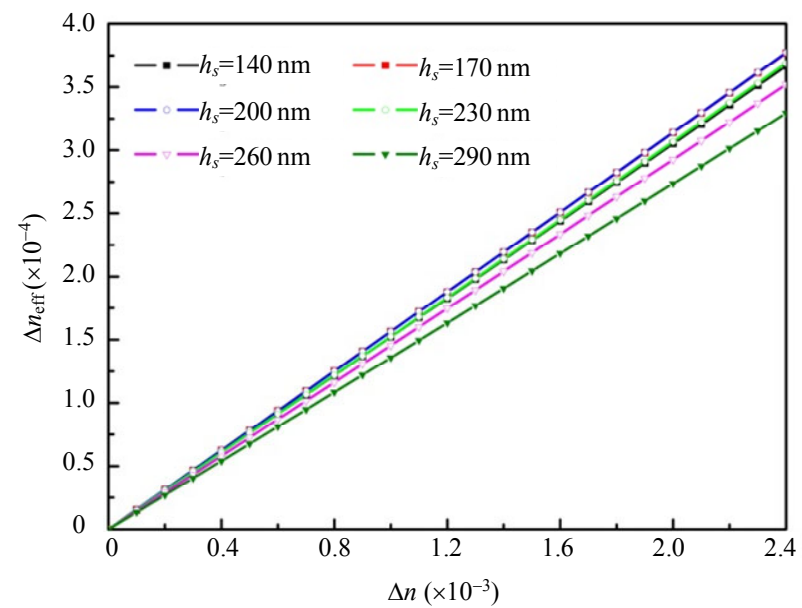

(b)

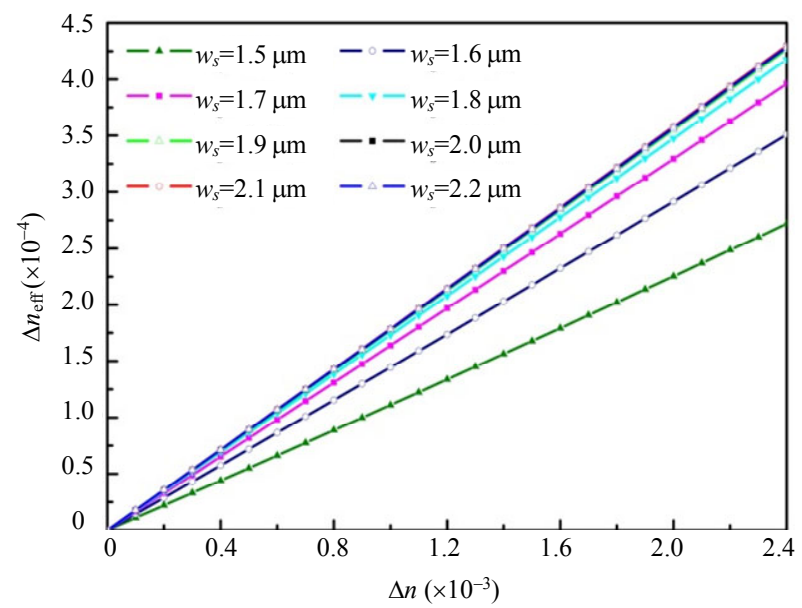

(d)

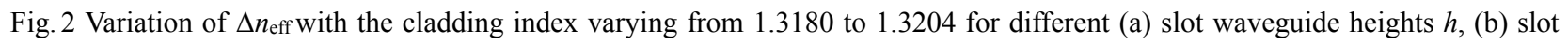
heights $h_{s}$, (c) height differences $\Delta h\left(=h_{2}-h_{1}\right)$ of the upper and the lower waveguide, and (d) waveguide widths $w_{s}$.

Since the height difference $\Delta h$ has a significant impact on the field distribution and hence the $n_{\text {eff, }}$ we also investigate the variation of $\Delta n_{\text {eff }}$ with $\Delta n$ for different $\Delta h$ while maintaining other parameters unchanged. The calculated results are shown in Fig. 2(c). It can be seen that the lines of $\Delta h=640 \mathrm{~nm}$ and $\Delta h=600 \mathrm{~nm}$ are total overlapped, which means both can realize the same change in effective index. Here $\Delta h=600 \mathrm{~nm}$ is used in our work, corresponding to $h_{1}=480 \mathrm{~nm}$ and $h_{2}=1080 \mathrm{~nm}$ in considering $h_{1}+$ $h_{2}=1.56 \mu \mathrm{m}$.

Finally, the influence of the waveguide width $w_{s}$ on $\Delta n_{\text {eff }}$ is also investigated when other optimal parameters are fixed, and the results with $w_{s}$ changing from $1.5 \mu \mathrm{m}$ to $2.2 \mu \mathrm{m}$ are shown in Fig. 2(d). It indicates that $\Delta n_{\text {eff }}$ reaches an almost identical maximum value when $w_{s}=2.0 \mu \mathrm{m}, 2.1 \mu \mathrm{m}$, and $2.2 \mu \mathrm{m}$, thus the waveguide width $w_{s}$ is set to be 
$2.1 \mu \mathrm{m}$, corresponding to the fabrication tolerances in width of $\pm 100 \mathrm{~nm}$.

Summarizing the investigation above, the optimal dimensions of the proposed polymer slot waveguide are $h_{s}=200 \mathrm{~nm}, h_{1}=480 \mathrm{~nm}, h_{2}=1080 \mathrm{~nm}$, and $w_{s}=2.1 \mu \mathrm{m}$. With these parameters, the electronic field distributions of the proposed waveguide are simulated and shown in Fig.3, which reveals preliminarily that the designed slot waveguide can achieve high sensitivity as high optical intensity can be achieved in the slot region. Furthermore, the waveguide sensitivity $S_{w}$ is calculated, and the result of 0.1791 is obtained. Compared with the silicon or silicon nitride slot waveguide, our proposed polymer slot waveguide offers comparably large waveguide sensitivity but larger dimensions, simpler and lower fabrication costs, and a lager slot allowing the liquid to penetrate better.

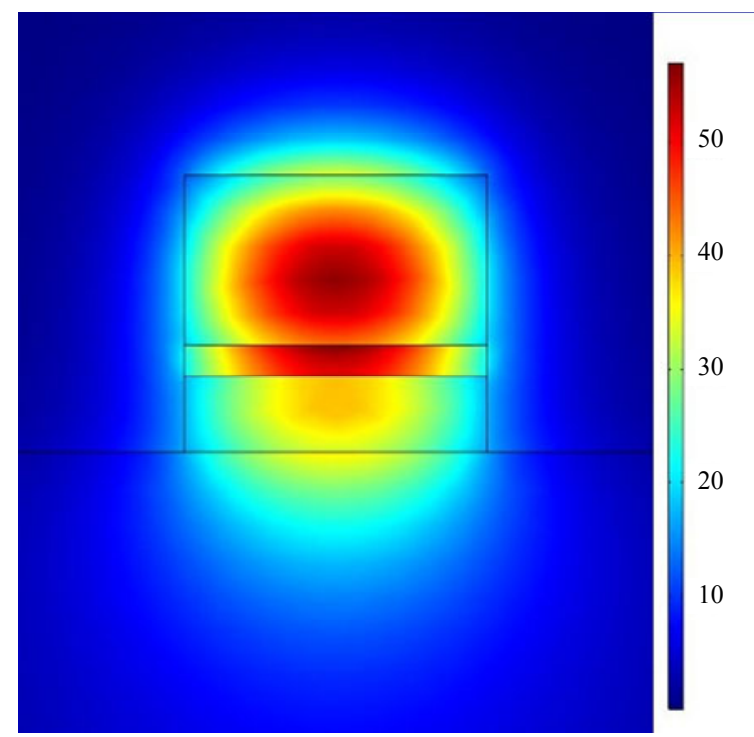

Fig. 3. Mode field distribution of the designed polymer horizontal slot waveguide.

\section{Sensor devices and theoretical results}

In order to evaluate the sensing performances of the polymer horizontal slot waveguide, sensors based on the MZI and the MRR configuration employing a polymer horizontal slot waveguide as a light-analyte interactive region are investigated. As a specific concentration of liquid corresponds to a specific refractive index, a change in liquid concentration leads to a change in the refractive index of liquid, and hence a change in the effective refractive index of waveguide, and at last a shift in the transmission spectrum of the MZI or MRR sensor. Therefore, the liquid concentration can be determined by measuring the shift in the transmission spectrum. The sensitivity of the device can be defined as [23]

$$
S=\frac{\partial \lambda}{\partial n}=\frac{\partial \lambda}{\partial n_{\text {eff }}} \cdot \frac{\partial n_{\mathrm{eff}}}{\partial n}=\frac{\partial \lambda}{\partial n_{\mathrm{eff}}} \cdot S_{w}
$$

where $\lambda$ is the operating wavelength, and $\partial \lambda / \partial n_{\text {eff }}$ is the wavelength shift caused by the sensor when the effective refractive index of the sensing waveguide is changed. The sensor sensitivity $S$ is an important parameter for evaluating the performances of sensor, and another important parameter is the limit of detection (LOD), which is defined by [4]

$$
L O D=\lambda_{\text {resolution }} / S
$$

where $\lambda_{\text {resolution }}$ is the wavelength resolution of the laser source.

\subsection{MZI sensor}

The proposed MZI sensor is shown schematically in Fig. 4. The optimized polymer horizontal slot waveguide with a length $L$ is inserted between two conventional polymer $S$ bent waveguides as sensing arm to realize light-analyte interaction. The reference arm, the $S$ bent waveguides, and the input and output waveguides are conventional polymer strip waveguide. A two-mode interference (TMI) is used to distribute more light power to the sensing arm so as to improve the extinction ratio in considering a larger propagation loss in the sensing arm than that in the reference arm and the notable coupling loss between the conventional strip waveguide and the slot waveguide. As shown in the inset of Fig. 4, the conventional strip waveguide and the slot waveguide have identical width $w_{s}(=2.1 \mu \mathrm{m})$ and 
height $h(=1.76 \mu \mathrm{m})$, and the cladding material of the conventional channel waveguide is polymethyl methacrylate (PMMA) with a refractive index of 1.4710 at $1538 \mathrm{~nm}$ for the TM polarization, where PMMA also has negligible material dispersion in the $\mathrm{C}$ band. To lower coupling loss between the fiber and input (output) waveguide, two wider strip waveguides are used as the input and the output waveguide, which are connected to the input and output ends of the MZI via two tapered strip waveguide, respectively.

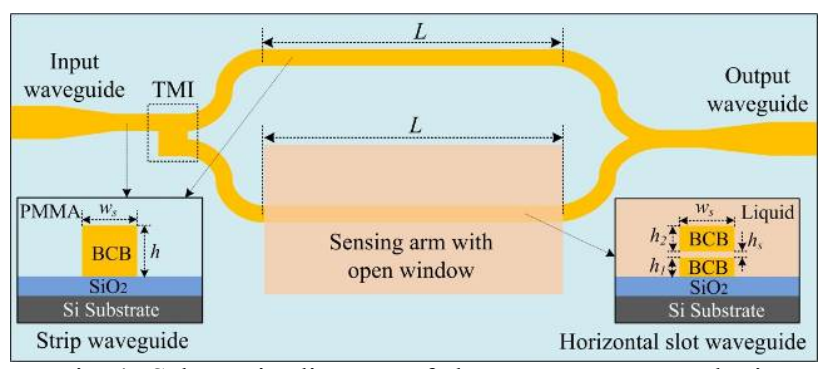

Fig. 4. Schematic diagram of the MZI sensor employing a polymer horizontal slot waveguide in one arm as light-analyte interactive region. Inset: cross-sectional view of the conventional strip waveguide (left) and the horizontal slot waveguide (right).

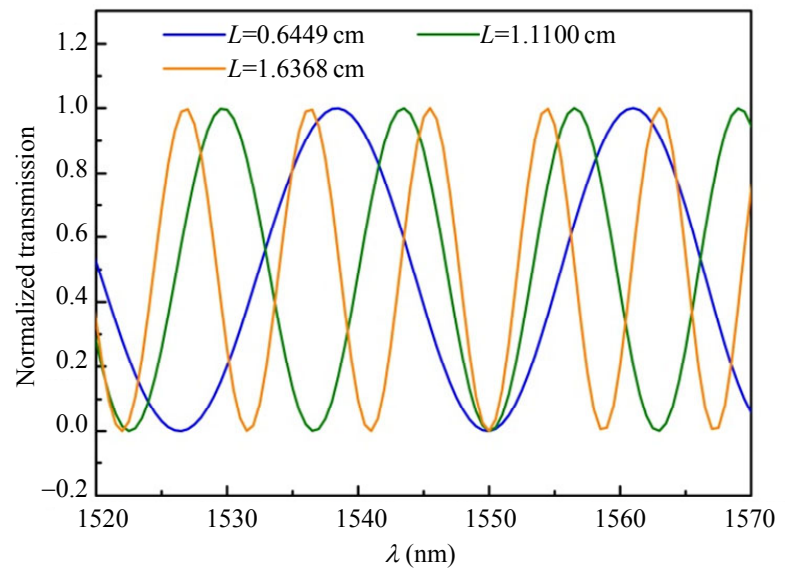

Fig. 5. Transmission spectra of the MZI sensor with $L=$ $0.6449 \mathrm{~cm}, 1.1100 \mathrm{~cm}$, and $1.6368 \mathrm{~cm}$.

According to the operating principle of MZI, the sensitivity $S$, i.e., the wavelength shift $\Delta \lambda$ in the interference fringes of the MZI, can be expressed as $S=\Delta \lambda=2 S_{w} L$. Obviously, the larger the length $L$ is, the higher the sensitivity $S$ is, but the larger the size of the device is. Therefore, it is necessary to choose a suitable length $L$ so as to achieve a high sensitivity with a reasonable size. For this purpose, the transmission spectra of the MZI sensor are simulated for different $L$ of $0.6449 \mathrm{~cm}, 1.1100 \mathrm{~cm}$, and $1.6368 \mathrm{~cm}$ in the range from $1520 \mathrm{~nm}$ to $1570 \mathrm{~nm}$, and the results are plotted in Fig. 5. As a trade-off between the size and sensitivity of the sensor, $L$ is set to be $1.1100 \mathrm{~cm}$.

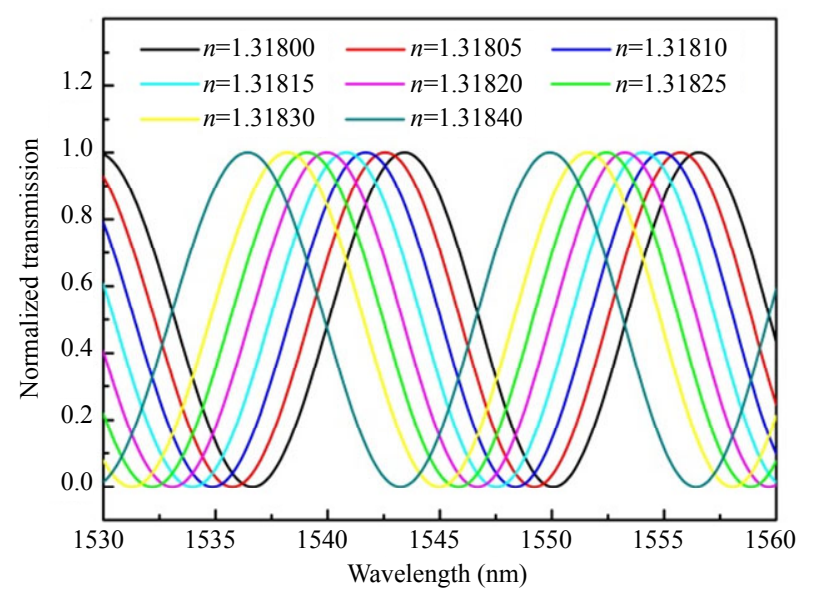

Fig. 6. Transmission spectra of the designed MZI sensor with $L=1.1100 \mathrm{~cm}$ for different refractive indices of the tested liquid.

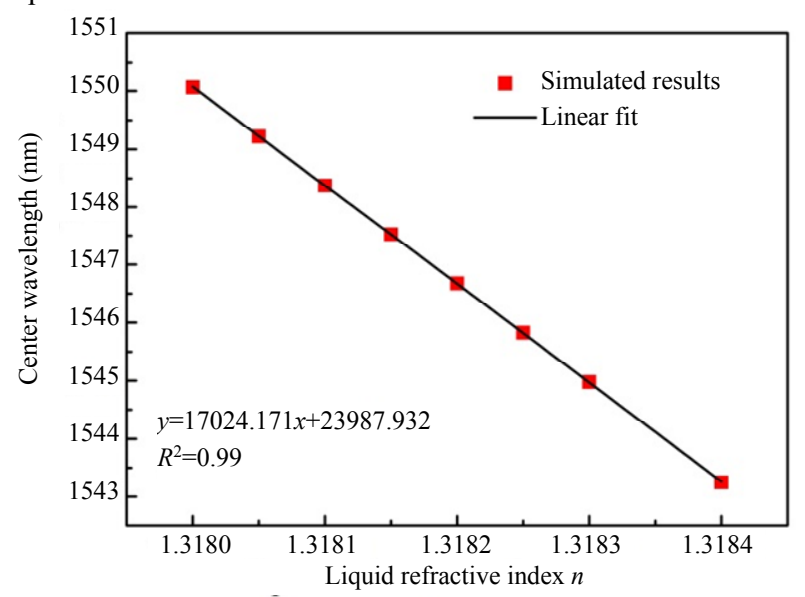

Fig. 7. Central wavelength versus refractive index of the tested liquid, and linear fit are used to calculate the sensitivity of the designed MZI sensor.

The normalized transmission spectra of the designed MZI sensor with 1.1100-cm-long sensing arm are simulated for different refractive indices of the tested liquid, and the results are shown in Fig. 6. It can be seen that the central wavelength of the MZI sensor decreases with an increase in the refractive index. The central wavelength versus refractive index of the tested liquid together with the 
linear fitting line is shown in Fig. 7. From the linear fitting line, the sensitivity of the designed MZI sensor is $17024 \mathrm{~nm} / \mathrm{RIU}$. The LOD of the designed MZI sensor is also calculated by considering $\lambda_{\text {resolution }}=0.03 \mathrm{~nm}$ of the spectrometer (Anritsu MS9740A), and the result is $1.76 \times 10^{-6} \mathrm{RIU}$, which is less than a third of that reported in [7]. For comparison, the phase change of the designed MZI sensor is also calculated at $1550 \mathrm{~nm}$ wavelength, and the result is $2311 \pi /(\mathrm{cm} \cdot \mathrm{RIU})$, which is slightly less than that of the MZI sensor based on the silicon nitride slot waveguide in [6]. In summary, the designed MZI employing polymer horizontal slot waveguide has a comparable sensitivity in comparison with the MZI sensor based on the conventional silicon or silicon nitride slot waveguide, even though the proposed polymer horizontal slot waveguide has a lower refractive index contrast.

\subsection{Microring resonant sensor}

The proposed MRR sensor is shown schematically in Fig. 8. The ring used in this work is a racetrack structure formed with two straight waveguides with length $L_{1}$ sandwiched between two semicircle waveguides with radius $r$. The ring is coupled to a straight waveguide with a gap $d$. The shaded region of the ring represents the sensing area with open window, where the polymer horizontal slot waveguide is used as a light-analyte interactive region while the conventional strip waveguide is used as a bracing structure, both labeled with different colors. Except for the rectangular waveguide in the center of ring, the dimensions of other strip waveguide, as shown in the inset of Fig. 8, are the same as the optimized slot waveguide so as to realize better physical connection and decrease the loss introduced by the bracing waveguide. The cladding material is also the PMMA. The total length of the ring is calculated by

$$
L_{\text {ring }}=2 \pi r+2 L_{1} \text {. }
$$

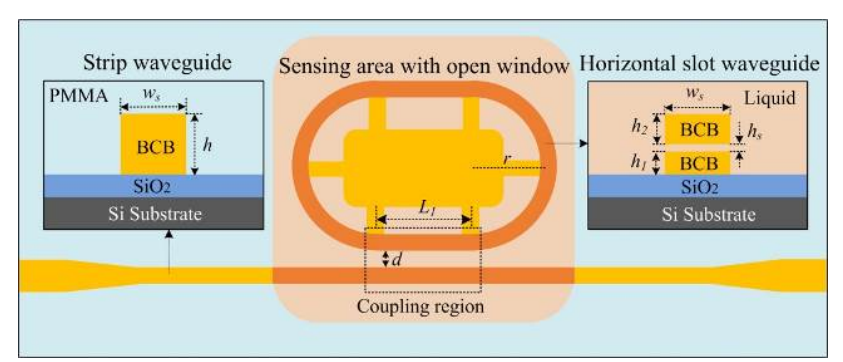

Fig. 8. Schematic diagram of the MRR sensor employing a polymer horizontal slot waveguide in the racetrack waveguide as light-analyte interactive region. Inset: cross-sectional view of the conventional strip waveguide (left) and the horizontal slot waveguide (right).

The transmission characteristics of a ring resonator is given by [24]

$$
T(\lambda)=\frac{a^{2}+t^{2}-2 a t \cos \left(\frac{2 \pi}{\lambda} n_{\mathrm{eff}} L_{\mathrm{ring}}\right)}{1+a^{2} t^{2}-2 a t \cos \left(\frac{2 \pi}{\lambda} n_{\mathrm{eff}} L_{\mathrm{ring}}\right)}
$$

where $n_{\text {eff }}$ is the effective index of guided mode in the ring, $a=\exp \left(-\alpha L_{\text {ring }}\right)$ is the loss factor in the ring with a waveguide loss coefficient of $\alpha$, and $t$ is the self-coupling coefficient in the coupling region. Just like the MZI sensor, the larger the radius $r$ of the MRR is, the higher the sensitivity $S$ of the sensor is, but the larger the size of the device is. Therefore, as an example, we choose $r=100 \mu \mathrm{m}$ and assume $a=$ 0.8 by considering the loss of ring. Here, to realize the maximum extinction ratio, the value of $t$ is set to equal to $a(t=a)$ [19], then, the coupling length $L_{1}=$ $125 \mu \mathrm{m}$ can be calculated when $d=1 \mu \mathrm{m}$ is fixed.

The calculated normalized transmission spectra of the proposed MRR sensor with the parameters above for different refractive indices of the tested liquid are shown in Fig. 9(a). The free spectral range (FSR) is about $1.8 \mathrm{~nm}$. Obviously, the transmission spectrum shifts toward a longer wavelength with an increase in the refractive index of the tested liquid. This is because when the refractive index of the tested liquid increases, the effective refractive index of the guided mode in the MRR will increase, resulting in an increase in the phase $\left(2 \pi n_{\text {eff }} L_{\text {ring }} / \lambda\right)$, consequently, the resonant wavelength will shift toward a longer wavelength.

Figure 9(b) shows the enlarged transmission spectrum in the dashed frame in Fig.9(a). From this 
figure, the relationship between the resonant wavelengths versus refractive index of the tested

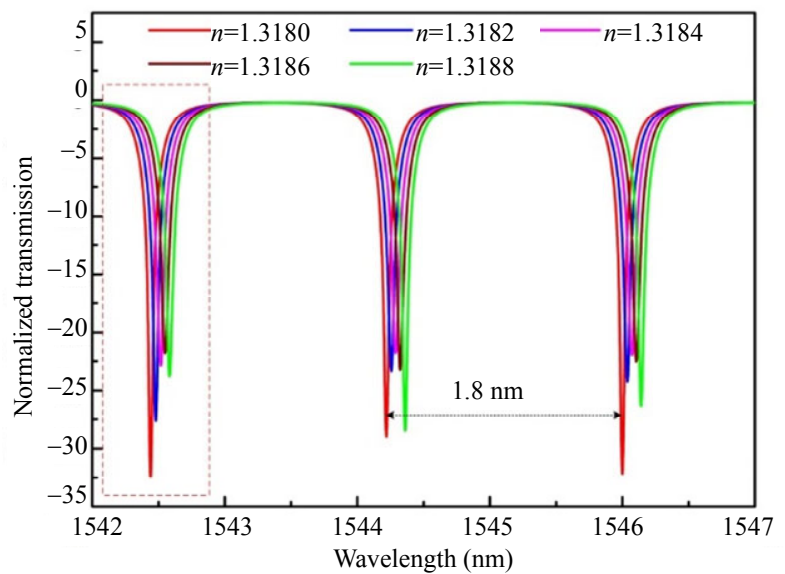

(a)

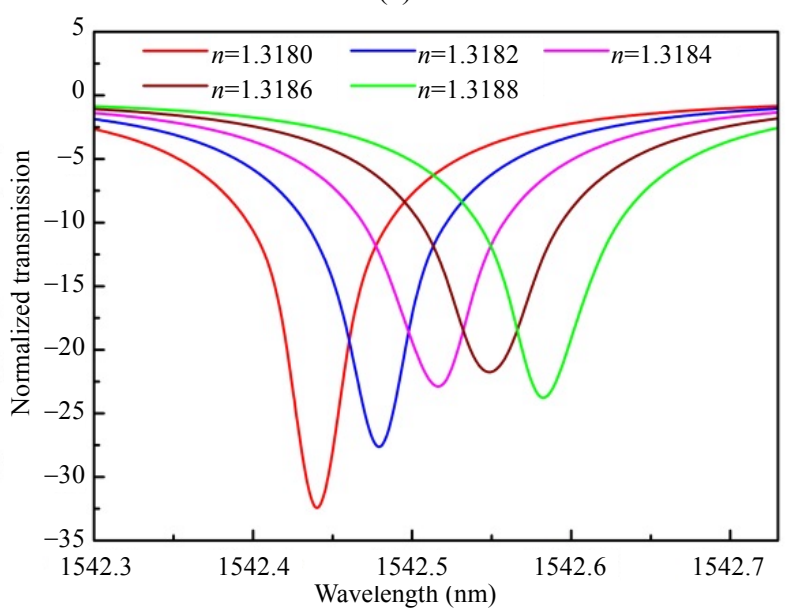

(b)

Fig. 9. Calculated normalized transmission spectra (a) and enlarged transmission spectra in the dashed frame (b) of the designed MRR sensor for different refractive index of the tested liquid with $r=100 \mu \mathrm{m}$ and $L_{l}=125 \mu \mathrm{m}$.

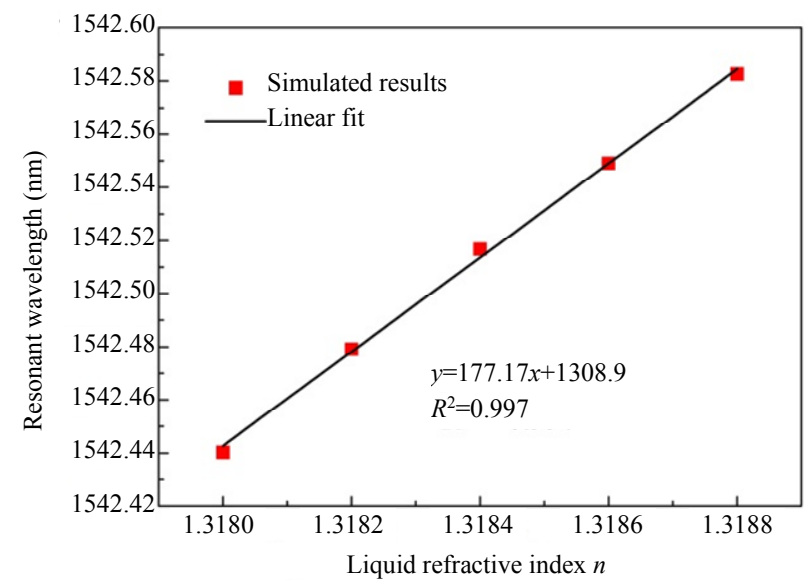

Fig. 10. Resonant wavelength versus refractive index of the tested liquid, and linear fittings are used to calculate the sensitivity of the sensor. liquid can be obtained easily and is shown in Fig. 10. The linear fitting line based on the calculated results is also shown in Fig. 10, which exhibits that the sensitivity of the designed MRR sensor is about $177 \mathrm{~nm} / \mathrm{RIU}$ and the corresponding LOD is $1.69 \times 10^{-4} \mathrm{RIU}$ with $\lambda_{\text {esolution }}=0.03 \mathrm{~nm}$. These values are somewhat smaller than that of a vertically slot-waveguide MRR sensor on a $\mathrm{Si}_{3} \mathrm{~N}_{4}-\mathrm{SiO}_{2}$ platform in which the sensitivity of $298 \mathrm{~nm} / \mathrm{RIU}$ [5] and $212 \mathrm{~nm} / \mathrm{RIU}$ and LOD of $\sim 2.3 \times 10^{-4}$ RIU [4] have been demonstrated. This can be attributed to the polymer slot waveguide having a much smaller refractive index difference.

\section{Conclusions}

A low-cost and highly sensitive liquid refractive index photonic sensor can be implemented by employing a polymer horizontal slot waveguide as light-analyte interactive region. Detailed analysis exhibits that our designed MZI- and MRR-based sensors with the optimized polymer horizontal slot waveguide can achieve the sensitivity of $17024 \mathrm{~nm} / \mathrm{RIU}$ and $177 \mathrm{~nm} / \mathrm{RIU}$ and the LOD of $1.76 \times 10^{-6} \mathrm{RIU}$ and $1.69 \times 10^{-4} \mathrm{RIU}$, respectively, with $\lambda_{\text {resolution }}=0.03 \mathrm{~nm}$. These results reveal that the sensor based on polymer horizontal slot waveguide can achieve comparable performances but with simpler and lower fabrication costs and lower scattering loss in interfaces in comparison with the sensor based on the silicon or silicon nitride slot waveguide. In addition, although the performances of the proposed MZI sensor are better than those of the proposed MRR sensor, the latter has a much smaller footprint than the former.

\section{Acknowledgment}

This work is supported by the National Natural Science Foundation of China (NSFC) (Grant No. 61505020) and the Opened Fund of the State Key Laboratory of Integrated Optoelectronics (Grant No. IOSKL2018KF12).

Open Access This article is distributed under the terms of the Creative Commons Attribution 4.0 International 
License (http://creativecommons.org/licenses/by/4.0/), which permits unrestricted use, distribution, and reproduction in any medium, provided you give appropriate credit to the original author(s) and the source, provide a link to the Creative Commons license, and indicate if changes were made.

\section{References}

[1] V. R. Almeida, Q. Xu, C. A. Barrios, and M. Lipson, "Guiding and confining light in void nanostructure," Optics Letters, 2004, 29(11): 1209-1211.

[2] Q. Xu, V. R. Almeida, R. R. Panepucci, and M. Lipson, "Experimental demonstration of guiding and confining light in nanometer-size low-refractiveindex material," Optics Letters, 2004, 29(14): $1626-1628$.

[3] C. A. Barrios, "Optical slot-waveguide based biochemical sensors," Sensors, 2009, 9(6): 47514765

[4] C. A. Barrios, K. B. Gylfason, B. Sánchez, A. Griol, H. Sohlström, M. Holgado, et al., "Slot-waveguide biochemical sensor," Optics Letters, 2007, 32(21): 3080-3082.

[5] T. Claes, J. G. Molera, K. D. Vos, E. Schacht, R. Baets, and P. Bienstman, "Label-free biosensing with a slot-waveguide-based ring resonator in silicon on insulator," IEEE Photonics Journal, 2009, 1(3): 197-204.

[6] Q. Liu, X. Tu, K. W. Kim, J. S. Kee, Y. Shin, K. Han, et al., "Highly sensitive Mach-Zehnder interferometer biosensor based on silicon nitride slot waveguide," Sensors and Actuators B: Chemical, 2013, 188(11): 681-688.

[7] M. Hiltunen, J. Hiltunen, P. Stenberg, S. Aikio, L. Kurki, K. Vahimaa, et al., "Polymeric slot waveguide interferometer for sensor applications," Optics Express, 2014, 22(6): 7229-7237.

[8] X. Wang, S. Grist, J. Flueckiger, N. A. F. Jaeger, and L. Chrostowski, "Silicon photonic slot waveguide Bragg gratings and resonators," Optics Express, 2013, 21(16): 19029-19039.

[9] V. M. Passaro, F. Dell'Olio, C. Ciminelli, C. Ciminelli, and M. N. Armenise, "Efficient chemical sensing by coupled slot SOI waveguides," Sensors, 2009, 9(2): 1012-1032.

[10] C. A. Barrios, B. Sánchez, K. B. Gylfason, A. Griol, H. Sohlström, M. Holgado, et al., "Demonstration of slot-waveguide structures on silicon nitride / silicon oxide platform," Optics Express, 2007, 15(11): 6846-6856.

[11] A. Spott, T. Baehrjones, R. Ding, Y. Liu, R. Bojko, T. O'Malley, et al., "Photolithographically fabricated low-loss asymmetric silicon slot waveguides," Optics Express, 2011, 19(11): 10950-10958.

[12] F. Dell'Olio and V. M. N. Passaro, "Optical sensing by optimized silicon slot waveguides," Optics Express, 2007, 15(8): 4977-4993.
[13] C. Viphavakit, M. Komodromos, C. Themistos, W. S. Mohammed, K. Kalli, and B. M. A. Rahman, "Optimization of a horizontal slot waveguide biosensor to detect DNA hybridization," Applied Optics, 2015, 54(15): 4881-4888.

[14] S. Lee, S. C. Eom, J. S. Chang, C. Huh, G. Y. Sung, and J. H. Shin, "Label-free optical biosensing using a horizontal air-slot SiNx microdisk resonator," Optics Express, 2010, 18(20): 20638-20644.

[15] P. T. Lin, S. Kwok, H. Y. G. Lin, V. Singh, L. C. Kimerling, G. M. Whitesides, et al., "Mid-infrared opto-nanofluidic slot-waveguide for label-free on-chip chemical sensing," Nano Letters, 2014, 14(1): 231-238.

[16] B. Kumaria, R. K. Varshneya, and B. P. Pal, "Design of chip scale silicon rib slot waveguide for sub-ppm detection of $\mathrm{N}_{2} \mathrm{O}$ gas at mid-ir band," Sensors and Actuators B: Chemical, 2018, 255: 3409-3416.

[17] L. Wang, J. Ren, X. Han, T. Claes, X. Jian, P. Bienstman, et al., "A label-free optical biosensor built on a low-cost polymer platform," IEEE Photonics Journal, 2012, 4(3): 920-930.

[18] C. Y. Chao, W. Fung, and L. J. Guo, "Polymer microring resonators for biochemical sensing applications," IEEE Journal of Selected Topics in Quantum Electronics, 2006, 12(1): 134-142.

[19] J. Halldorsson, N. B. Arnfinnsdottir, A. B. Jonsdottir, B. Agnarsson, and K. Leosson, "High index contrast polymer waveguide platform for integrated biophotonics," Optics Express, 2010, 18(15): 16217-16226.

[20] J. Chovan and F. Uherek, "Polymeric slot waveguide for photonics sensing," in 20th Slovak-Czech-Polish Optical Conference on Wave and Quantum Aspects of Contemporary Optics, Jasna, Slovakia, 2016, 10142, pp. 101420P-1-101420P-7.

[21] G. M. Hale and M. R. Querry, "Optical constants of water in the $200-n m$ to $200-\mu \mathrm{m}$ wavelength region," Applied Optics, 1973, 12(3): 555-563.

[22] A. F. Fucaloro, Y. Pu, K. Cha, A. Williams, and K. Conrad, "Partial molar volumes and refractions of aqueous solutions of fructose, glucose, mannose, and sucrose at $15.00,20.00$, and $25.00^{\circ} \mathrm{C}$," Journal of Solution Chemistry, 2007, 36(1): 61-80.

[23] X. Sun, D. Dai, L. Thylén, and L. Wosinski, "High-sensitivity liquid refractive-index sensor based on a Mach-Zehnder interferometer with a double-slot hybrid plasmonic waveguide," Optics Express, 2015, 23(20): 25688-25699.

[24] S. Chandran, K. Ramesh, and B. K. Das, "Dispersion enhanced critically coupled ring resonator for wide range refractive index sensing," IEEE Journal of Selected Topics in Quantum Electronics, 2016, 23(2): 424-432.

[25] W. Zhang, S. Serna, X. L. Roux, L. Vivien, and E. Cassan, "Highly sensitive refractive index sensing by fast detuning the critical coupling condition of slot waveguide ring resonators," Optics Letters, 2016, 41(3): 532-535. 